\title{
Study by Inverse Method the Size Distribution of the Particle of Carbonaceous Generated in RF Discharge
}

\author{
A. El Kebch ${ }^{*}$, M. El Mouden'², N. Dlimi ${ }^{3}$, D. Saifaoui ${ }^{3}$ \\ ${ }^{1}$ Laboratory of Structural Engineering, Intelligent Systems and Electric Energy, National School of Arts and Crafts (ENSAM \\ Casablanca), Hassan II University Casablanca, Casablanca, Morocco \\ ${ }^{2}$ Laboratory of Engineering Sciences for Energy (LabSIPE), University Research Center (CUR) in Renewable Energies \& Intelligent \\ Systems for Energy EnR\&SIE, National School of Applied Sciences, Chouaib Doukkali University, El Jadida, Morocco \\ ${ }^{3}$ Laboratory of Theoretical and Applied Physics, Faculty of Sciences Ain Chock, Casablanca Hassan II University Casablanca, \\ Casablanca, Morocco \\ Email: *ALI.ELKEBCH@univh2c.ma, *alibec_ma@yahoo.fr
}

How to cite this paper: El Kebch, A., El Mouden, M., Dlimi, N. and Saifaoui, D. (2019) Study by Inverse Method the Size Distribution of the Particle of Carbonaceous Generated in RF Discharge. Optics and Photonics Journal, 9, 99-111. https://doi.org/10.4236/opj.2019.97010

Received: March 12, 2019

Accepted: July 19, 2019

Published: July 22, 2019

Copyright (C) 2019 by author(s) and Scientific Research Publishing Inc. This work is licensed under the Creative Commons Attribution International License (CC BY 4.0).

http://creativecommons.org/licenses/by/4.0/

\begin{abstract}
One of the difficulties encountered in the study of dusty plasmas is related to the knowledge of the size of the dust particles present. A variety of sources, physical and chemical mechanisms of formation, causes a wide variety of sizes and morphologies of dust. The diameter of a dust will not be unique but spread over several orders of magnitude. Its distribution in number, surface, mass or volume is called distribution. It is important to know this distribution in particle size because it strongly impacts the physical and radiative processes. To have a dust distribution in situ is very difficult; the reverse method can identify the particle populations from light extinction measures. In this study, we present an inversion procedure with a Tikhonov regularization dedicated to the determination of volume size distribution (V-PSD) from extinction measurements corresponding to the different wavelengths obtained by the Extinction Spectrometry technique.
\end{abstract}

\section{Keywords}

Particle Size Distribution, Dust Plasma, Light Extinction Spectroscopy Measurements, Mie theory, Inversion, Tikhonov Regularization

\section{Introduction}

Dusty plasmas are the objects of intense research since the beginning of the years 1980. They are met in several fields such as astrophysics [1] [2] or Mi- 
cro-electronics industry using the processes plasma [3]. In addition, the researches undertaken in the context of the thermonuclear fusion controlled by magnetic containments reveal that important quantities of dust are produced within the plasma reactors. The formation of these dust results from the erosion of the plasma reactor walls by physical sputtering [4], by chemical erosion or by melting/sublimation consecutive to an abnormal event. These dusts can pollute the confined plasma and play a critical role in the effectiveness of the plasma discharge by causing significant losses by radiation. In addition, these dusts can be a health problem especially when they contain tritium [5] [6] [7].

Due to the importance of these topics, in particular in the framework of the development of the international reactor ITER, many experimental works have been performed for many years to understand the growth mechanisms and the associated dynamics of these dusts [8]. To study these two last variables, the measurements of the size distribution and the concentration of particles flowing in dusty reactive plasma are really relevant. But for that, these measurements need to be non-intrusive, realized within the plasma reactor as well as being spatially and temporally resolved. This is why the technique referenced as "Light Extinction Spectroscopy (LES)" seems to be the most appropriate for dust assessments in fusion device reactors.

An incident radiation that propagates in a vacuum, undergoes no modification either in its intensity or its state of polarization [9]. An electromagnetic wave that propagates in a medium of electric charges will cause the oscillation of the charges, so a part of the energy of the wave is absorbed as an oscillating electric charge radiates. This absorbed energy is re-put into the medium in the form of an electromagnetic wave propagating in a new direction.

This diffusion results in [10]:

- Modification of the direction of propagation of the incident wave;

- The absorption that will characterize the decrease of the energy of the incident wave.

It is therefore interesting to note that the diffusion phenomenon is related to the nature of the propagation medium and depends on the geometry of the particles of the medium.

Due to the importance of the subject dusty plasma and to understand the mechanisms of growth of these dusts, as well as their dynamics, many experimental works have been carried out for many years. The analysis and the knowledge of the particle size of the dust in the plasma make it possible to better understand the behavior of the dust.

These small particles have been of particular scientific interest, Given the wide variety of applications in materials technologies, and in the electronics and optics field. A promising technique for accurate real-time and in-situ measurement of the size and concentration of sets of nanoparticles is the extinction spectroscopy (TES) technique. This technique is based on measuring the transmission spectrum of a light that passes through a medium that contains particles (liquid or solid). The intensity of this transmitted light is a function of its wavelength, 
the size, the concentration and the refractive index of the particles. The spectrum of transmitted light is modeled using a light scattering theory, and a data inversion algorithm is used to determine the volume size distribution of the particles. The measurement technique has several advantages including limited optical access, the ability to perform long-range measurements, a simple optical configuration and a short measurement time. These advantages could make this technique widely used in industrial and scientific environments.

Unfortunately, the data inversion algorithm requires the resolution of a Fredholm equation of the first type, which is a well-known but poor resolution problem. This problem must be regularized to ensure that the determined size distribution has a physical meaning. A detailed literature exists regarding this technique. [11] [12] [13] [14].

In the work reported here, LES is used to characterize the growth of carbonaceous particles produced in an Argon RF discharge. After this short introduction, the experimental setups are described: the plasma reactor, the LES device as well as its principles and the inversion and regularization procedures developed to interpret the recorded experimental data. In the last part, experimental results are presented and discuted.

\section{Experimental Devices}

\subsection{Experimental Plasma Reactor [15]}

The reactor used in this experimental study is shown in Figure 1. It is composed of a cylindrical stainless steel chamber of $D_{e}=30 \mathrm{~cm}$ in diameter and $H_{e}=35 \mathrm{~cm}$ in height, and of two stainless steel electrodes of $L_{e}=5 \mathrm{~cm}$ : a cathode in its superior part and a grounded anode situated $h_{e}=8 \mathrm{~cm}$ below. The cathode is powered with a radio-frequency (RF) generator operating at $13.56 \mathrm{MHz}$ (Dressler Cesar 133). An automatic matching box placed between the generator and the chamber optimizes the power coupling. A removable stainless envelope protects the walls of the chamber of dust deposits. Eight glass portholes (KB7) situated halfway up are distributed around the chamber every 45 degrees. They can be replaced if necessary by quartz windows to allow measurements in the UV spectral range. A vacuum of less than $3-10$ torr, which is controlled by a capacitive gauge (Balzers TPG 252), is achieved using a vane pump (Leybold AS8-16) equipped with a dust filter. A mixture of argon and acetylene $\left(\mathrm{C}_{2} \mathrm{H}_{2}\right)$, whose quantities are controlled using mass flow meters, is injected within the chamber through micro-holes. In the present study, the mixture fraction in volume is fixed to $\mathrm{Y}_{\mathrm{C} 2 \mathrm{H} 2 / \mathrm{Ar}} \approx 43 \%$, $\left(\mathrm{Ar} 7 \mathrm{sccm}+\mathrm{C}_{2} \mathrm{H}_{2} 3 \mathrm{sccm}\right)$ while the static pressure of Argon and the electrical power are maintained respectively at $P=2.7610^{-1} \mathrm{mBar}$ and $P_{\text {elec }}=20 \mathrm{~W}$.

Because of its geometry and its operating mode, a large amount of carbonaceous particles, whose diameters range from tens to hundreds of nanometers, are trapped near the cathode. This is the result of a balance between several forces: the gravity force, electrostatic forces and the drag forces. 


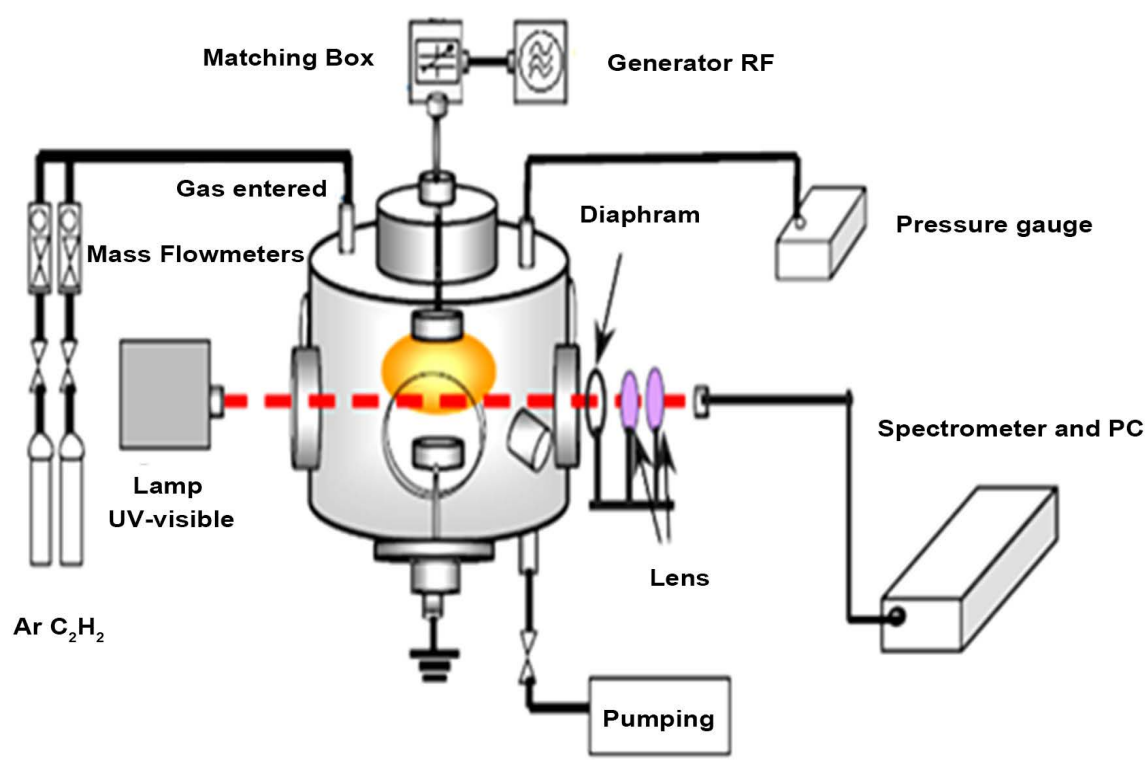

Figure 1. Sketch of the experimental devices.

This is this cloud of particles that is studied hereby light extinction spectroscopy (LES) to determine the particle size distributions (PSD) and the particle concentration near the cathode during the plasma ignition.

\subsection{Light Extinction Spectroscopy (LES)}

\subsubsection{LES Principle}

The LES technique consists in illuminating the dust to characterize by a collimated beam of polychromatic light [16]. If the collection of multi-scattered photons is negligible, the spectral transmission rate of the incident beam can be written:

$$
T\left(\lambda_{i}\right)=\frac{I\left(\lambda_{i}\right)}{I_{0}\left(\lambda_{i}\right)}=\exp (-\tau L)
$$

where $\lambda_{i}$ is the wavelength, $I_{0}\left(\lambda_{i}\right)$ and $I\left(\lambda_{i}\right)$ are the spectral intensities of the incident beam and the one after passing through the cloud of particles, $L$ the path length of the beam through the particulate media, $\tau=N \cdot C_{e x t}$ the turbidity, $N$ the particle number concentration, $C_{e x t}$ the mean extinction cross section. If we assume that particles are spherical with a diameter $D, C_{e x t}$ is an integral quantity depending of the extinction cross-section $Q_{\text {ext }}(D, \tilde{m}, \lambda)$ and the statistical weight of each particle in the cloud [17] [18]:

$$
C_{\text {ext }}=N \int_{D_{\min }}^{D_{\max }} Q_{\text {ext }}(D, \tilde{m}, \lambda) \frac{\pi D^{2}}{4} f(D) \mathrm{d} D
$$

where $\tilde{m}$ is the complex refractive index of each particle.

\subsubsection{Inversion Procedure}

From a mathematical point of view, the Equation (2) is an inhomogeneous Fredholm equation of the first kind. Our problem is to determine the concentra- 
tion $N$ of the dust and particle size distribution $f(D)$, from the measurement of $T\left(\lambda_{i}\right)$ and the theoretical knowledge of the kernel $Q_{\text {ext }}(D, \tilde{m}, \lambda)$. In fact, this problem is predetermined and ill-posed. Its resolution requires the development of a specific inversion technique. For numerical reasons, it is preferable to rewrite the Equation (2) with volume quantities rather than in number. For spherical particles, the dust volume concentration $C_{V}$ is simply related to the number concentration by:

$$
V(D)=C_{v} v(D)=N\left(\frac{\pi}{6}\right) D^{3} f(D)
$$

where $V(D)$ is the particle size distribution in volume and $v(D)$ the normalized particle size distribution in volume. If the number quantities are replaced by volume ones and by introducing the constant $\Lambda=-3 / 2 L$, the Equation (2) takes the form:

$$
\ln \left[T\left(\lambda_{i}\right)\right]=\Lambda \int_{D_{\min }}^{D_{\max }} Q_{\text {ext }}(D, \tilde{m}, \lambda) \frac{V(D)}{D} \mathrm{~d} D
$$

The previous integral equation can be discretized as follows [16]:

$$
\int_{D_{\min }}^{D_{\max }} Q_{e x t}(D, \tilde{m}, \lambda) \frac{V(D)}{D} \mathrm{~d}(D)=\sum_{j=1}^{M} S_{i j} V_{j}
$$

where $V(D)$ is the quantity we want to determine and that can be discretized by the vector $V_{j}$ with $j=1,2 \cdots, M,\left(S_{i j}\right)$ is the element of the "extinction matrix" whose dimensions are equal to $N \times M$. The latter is determined for a given wavelength $\lambda_{i}, \quad i=1, \cdots, N$ and a class of diameters ranging from $D_{j-1}$ et $D_{j}$

$$
S_{i j}=\int_{D_{j-1}}^{D_{j}} \frac{Q_{e x t}(D, \tilde{m}, \lambda)}{D} \mathrm{~d}(D)
$$

The measured transmission spectrum can also be written as a one-dimensional vector $T$ whose each elements $T_{i}$ represents the measured transmission for the wavelength $\lambda_{i}$ within a particles cloud. To determine the discretized PSD in volume $V$, we just have to solve the algebraic equation: $S V \equiv T$. This equation admits a trivial solution:

$$
V=\left(S^{t r} S\right)^{-1} S^{t r} T
$$

However to stabilize the inversion procedure, it is preferable to iteratively minimize the quantity $S V$-T with a least-square algorithm and a non negative constraint:

$$
\min \|S . V-T\|_{2-L S Q}^{2}
$$

The minimization procedure can be realized with orthogonal algorithms [19].

\subsubsection{Regularization of the Problem}

It has to be noted that the previous method has already been used successfully to inverse critical scattering patterns produced by clouds of bubbles. But in the argon-acetylene RF discharge studied here, the particles generated have a diameter 
ranging from ten to several hundred nanometers, i.e. ranging between the Rayleigh and Mie scattering regimes. This implies that the extinction coefficients of the cloud of particles probed can vary from several orders of magnitude from the lower to the upper boundaries of their associated PSDs. As a result the associated extinction matrix $S$ has a very high condition number, which requires the development of a specific regularization procedure to stabilize the inversion of LES measurements. Finally, the inversion procedure developed and used here is based on a least squares algorithm, associated with a Tikhonov regularization method [20] that performs the following minimization:

$$
\min _{V \succ 0}\left\{\|S . V-\bar{T}\|_{2-L S Q}^{2}+\gamma^{2}\left\|H\left(V-V_{0}\right)\right\|_{2-L S Q}^{2}\right\}
$$

where $H$ is a smoothing matrix that is here the identity matrix, $\gamma$ a regularization parameter whose optimal value is estimated by the method of L-Curve [21] [22], $V_{0}$ an assumed initial solution vector which is here chosen equal to zero.

\subsubsection{Experimental LES Device}

The plasma is illuminated by a stabilized UV-visible lamp (BAL DH2000-Deuterium Tungsten Halogen Light Source). The probing beam is collimated on the input porthole of the reactor by a system composed of two Plano-convex lenses, see Figure 1. Its diameter ranges from $3 \mathrm{~mm}$ to $10 \mathrm{~mm}$. Downstream the plasma reactor, a diaphragm is used to reduce the collection of the plasma emissions. Then the beam is collected through an optical fiber connected to a spectrometer (Ocean Optics).

The later was equipped with a CCD sensor of 2048 pixels (AvaSpec-2048), and has a spectral resolution of $0.8 \mathrm{~nm}$ (FWHM). It authorizes the acquisition of a complete spectrum in the wavelength range [200 nm; $1100 \mathrm{~nm}$ ] at relatively high frequencies. Here the integration time of each recording ranges from $100 \mathrm{~ms}$ to $200 \mathrm{~ms}$, with an acquisition rate fixed at $1 \mathrm{~Hz}$. whose spectral acquisition range is [300 nm; $1100 \mathrm{~nm}$ ].

The latter is connected to a PC which permits to control it, to record data or to inverse them.

\section{Experimental Results and Discussion}

\subsection{LES Measurement Protocols}

To perform LES measurements, the following experimental protocol has been used:

1) $\Delta t=0 \mathrm{~s}$, the LES lamp is ignited during $\Delta \mathrm{t}=120 \mathrm{~s}$ to stabilize its intensity,

2) $\Delta t=120 \mathrm{~s}$, a plasma made of pure argon is ignited during $\Delta \mathrm{t}=120 \mathrm{~s}$,

3) $\Delta \mathrm{t}=240 \mathrm{~s}$, acetylene is introduced during (a) $\Delta t=1200 \mathrm{~s}$ to perform a study of the spatial reparation of dust within the plasma reactor or during (b) $\Delta t$ $=3600 \mathrm{~s}$ to study the role of several plasma parameters on the formation of dusts,

4) $\Delta t=1440 \mathrm{~s}$, acetylene injection is stopped, but the plasma of argon is still 
ignited,

5) $\Delta t=1470 \mathrm{~s}$, the plasma of argon is off.

These five phases will be discussed later in the section «Effects of the distance relative to the cathode».

\subsection{Experimental LES Measurements}

Figure 2 presents the temporal evolution of the transmission recorded for three different vertical positions of the LES probing beam below the cathode: (a) $\Delta h_{1}=$ $0 \mathrm{~cm}, \Delta h_{2}=0.5 \mathrm{~cm}, \Delta h_{3}=1 \mathrm{~cm}$. In these three cases, the plasma operating conditions are given in Section 2.1.

Several commentaries can be made regarding the Figure 2:

- Whatever the wavelength for a given position, the temporal evolution of the transmission spectrum has the same shape. As already shown on Figure 2, the transmission rate is lower as the wavelength is low.

- More the LES measurements are made close to the cathode, more the transmission rate decreases. For example, for a wavelength of $\lambda=300 \mathrm{~nm}$, the transmission rate decreases from $70 \%$ at $\Delta h_{3}$, to $50 \%$ at $\Delta h_{2}$, to fall to $15 \%$ near the cathode (at $\Delta h_{1}$ ).

When the plasma is ignited and $\mathrm{C}_{2} \mathrm{H}_{2}$ is injected, i.e. during the phase III, we can notice that near the cathode (at $\Delta h_{1}$ ) and for a given wavelength, the temporal evolution of $T$ present several variations. Conversely far away from the cathode (at $\Delta h_{3}$ ), $T$ remains almost constant over the phase III, except during the first $100 \mathrm{~s}$ of this phase. It seems that the temporal variations of $T$ for a given wavelength are attenuated when the distance between the probed volume and the cathode increases. This may result from the movements of carbonaceous particles produces in this area.

\subsection{Inversion of LES Measurements}

\subsubsection{Inversion Parameters}

The space of the diameters particles is discretized in $M=100 \mathrm{c}$ lasses of $10 \mathrm{~nm}$ wide, distributed linearly between $D_{\min }=10 \mathrm{~nm}$ and $D_{\max }=1 \mu \mathrm{m}$. The spectral discretization is effected using $J=71$ wavelengths distributed between $\lambda_{\min }=300$ $\mathrm{nm}$ and $\lambda_{\min }=1000 \mathrm{~nm}$. The length of the probe volume is equal to $L=30 \mathrm{~cm}$, i.e. the reactor diameter.

\subsubsection{Effects of the Distance Relative to the Cathode}

Figure 3 shows the PSD in (noted V-PSD) volume obtained by LES measurements at the 3 positions described before and with the plasma operating conditions described in Section 2.1. Figure 3 indicates that near the cathode the particle size distribution is broader, it is observed that when approaching the cathode increases the density of the dusty, it is deduced that the powders are trapped near the cathode and are deposited on it during the discharge which is why the greater the distance of the cathode, more transmission rate approaches 1 (whatever the wavelength), more the density of the dust decreases. 


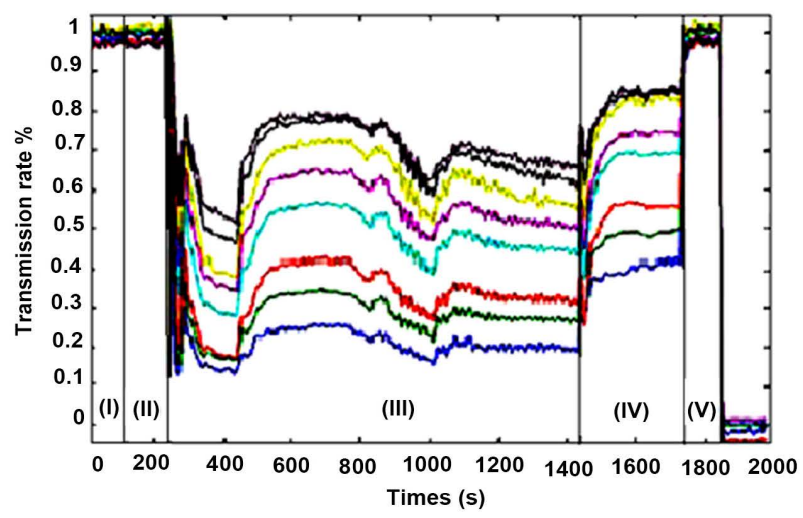

(a)

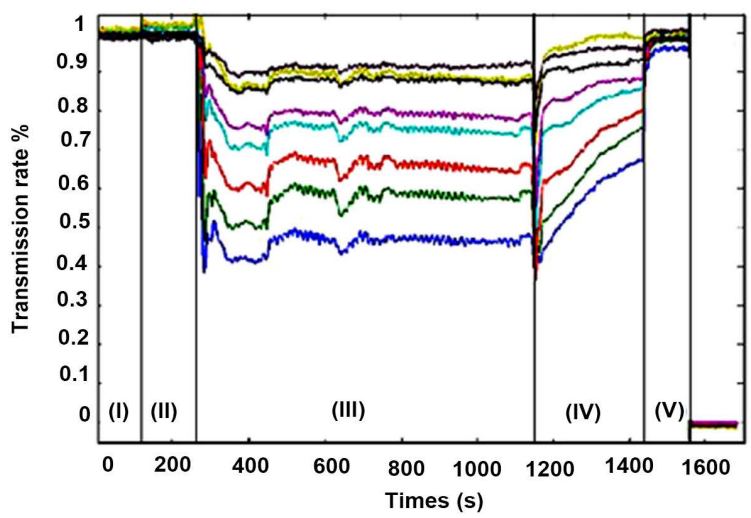

(b)

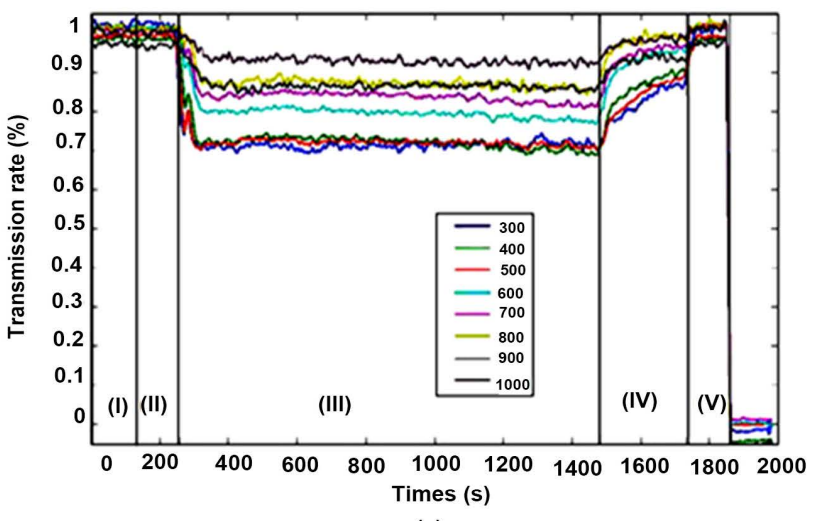

(c)

Figure 2. Time evolution of the transmission recorded in dusty plasma for three vertical positions below the cathode: (a) $\Delta h_{1}=0$ $\mathrm{cm}$, (b) $\Delta h_{2}=0.5 \mathrm{~cm},(\mathrm{c}) \Delta h_{3}=1 \mathrm{~cm} \mathrm{[15].}$

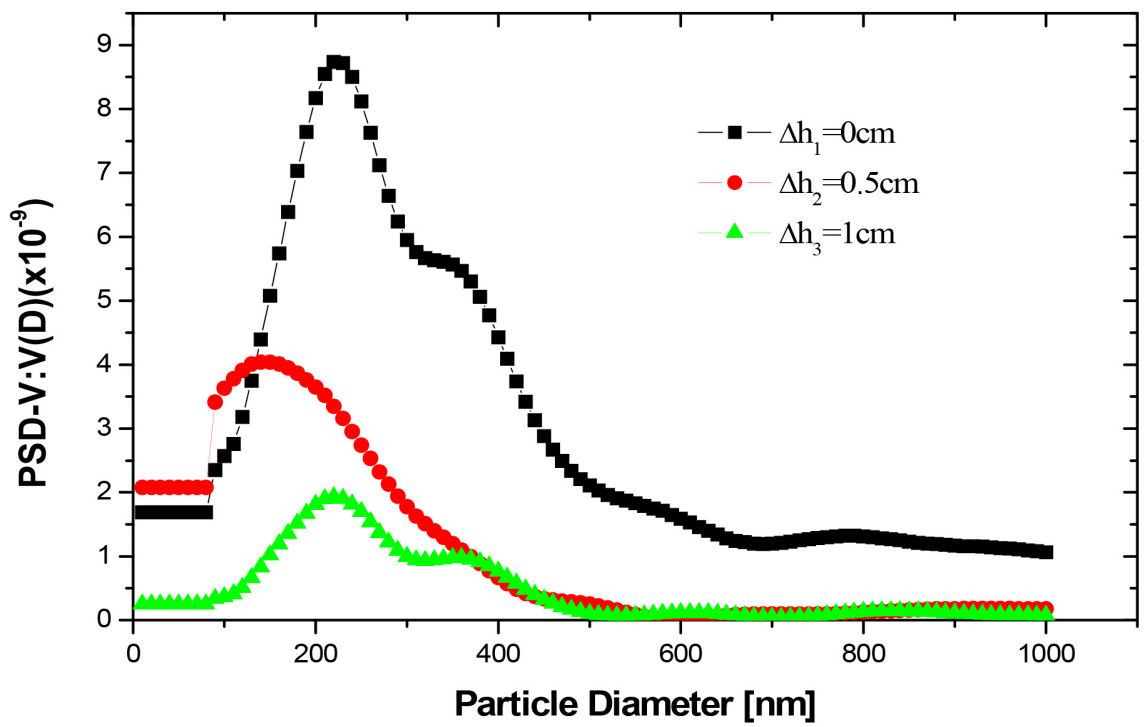

Figure 3. PSD measured at 3 different positions below the cathode $\left(\Delta h_{1}, \Delta h_{2}, \Delta h_{3}\right)$ at $\Delta t=$ $400 \mathrm{~s}$ after the beginning of the experiment, i.e. $\Delta t=160 \mathrm{~s}$ after the beginning of the powder generation.

\subsubsection{Effects of the Discharge Duration}

Figure 4 reports the temporal evolution of the mean diameter measured by LES 
at a distance of $\Delta h_{1}$ below the cathode. The value of the mean diameter in volume $D_{v}$ varies during experiment. It reaches its maximum value of $D_{\max }=390$ $\mathrm{nm}$ after $110 \mathrm{~s}$ of $\mathrm{C}_{2} \mathrm{H}_{2}$ injection. Then $D_{V}$ decreases after $\Delta t=200 \mathrm{~s}$, while its value oscillates between $\Delta t=360 \mathrm{~s}$ and $\Delta t=660 \mathrm{~s}$ and lastly increases after $\Delta t=$ $660 \mathrm{~s}$ of $\mathrm{C}_{2} \mathrm{H}_{2}$ injection. This evolution of $D_{v}$ is in good agreement with the temporal evolution of the transmission reported in the section «Experimental LES measurements». In addition, Figure 4 shows that the temporal evolution of the concentration in volume $C_{v}$ follows the one of $D_{r}$. This reveals that particles, which are produced near the cathode, move towards the anode between $\Delta t=360$ $\mathrm{s}$ and $\Delta t=660 \mathrm{~s}$ after the start of the $\mathrm{C}_{2} \mathrm{H}_{2}$ injection. Then $C_{v}$ increases as well as $D_{v}$ increases or oscillates. This may reveal the formation of a new generation of podwer.

\subsubsection{Effects of the Optical Index on the Granulometry}

The three main drawbacks of using the LES technique to study the growth of carbonaceous nano-powders produced in RF discharges are that 1) the composition of theses nano-powders is not known, 2) in addition their composition can vary during the plasma ignition, 3) lastly the inversion procedure of LES measurements requires to know the refractive index on a large spectral range. In a first approach, different refractive indices found in the literature can be used. But the effect of the choice of a refractive index over another on the LES inversion results must be estimated. Table 1 summarizes the 3 different refractive indices of carbonaceous particles tested in this work.

As might be expected, the choice of the refractive index used to inverse LES measurement modifies the reconstructed PSD (see Figure 5). The associated concentration in volume is reported on Table 2. It appears that less the material constituting the nano-podwers is absorbent, more the concentration in volume is higher.

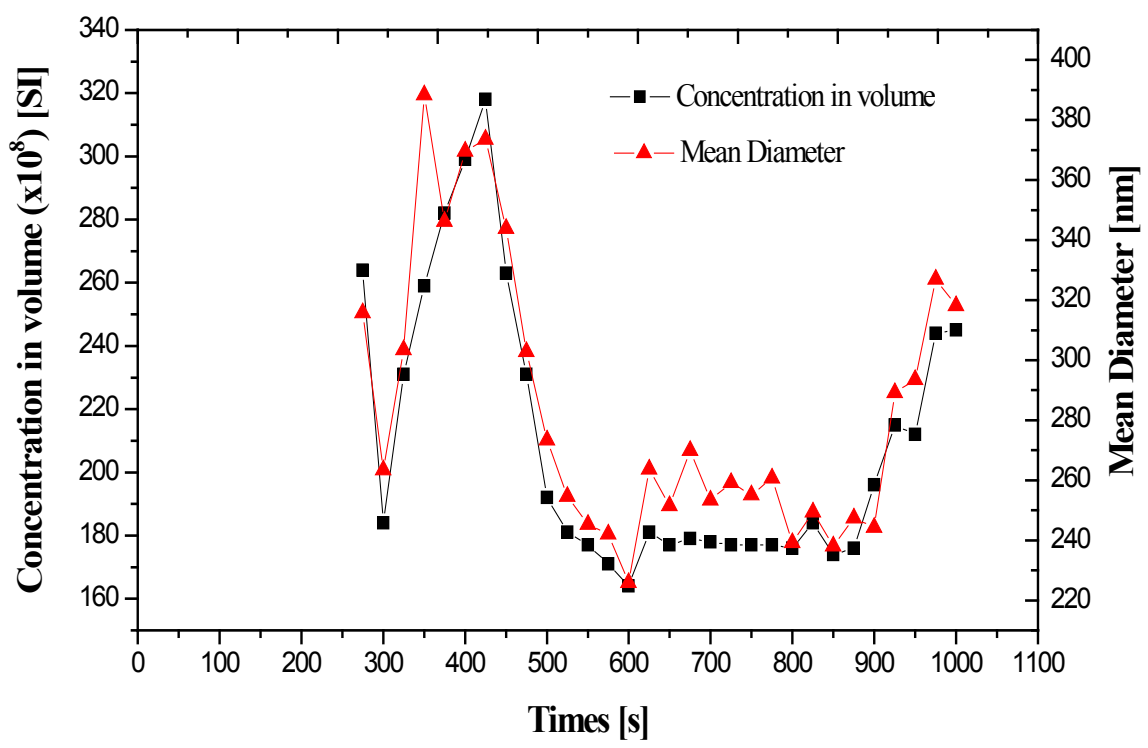

Figure 4. Effects of the discharge duration $\left(\Delta h_{1}\right)$. 


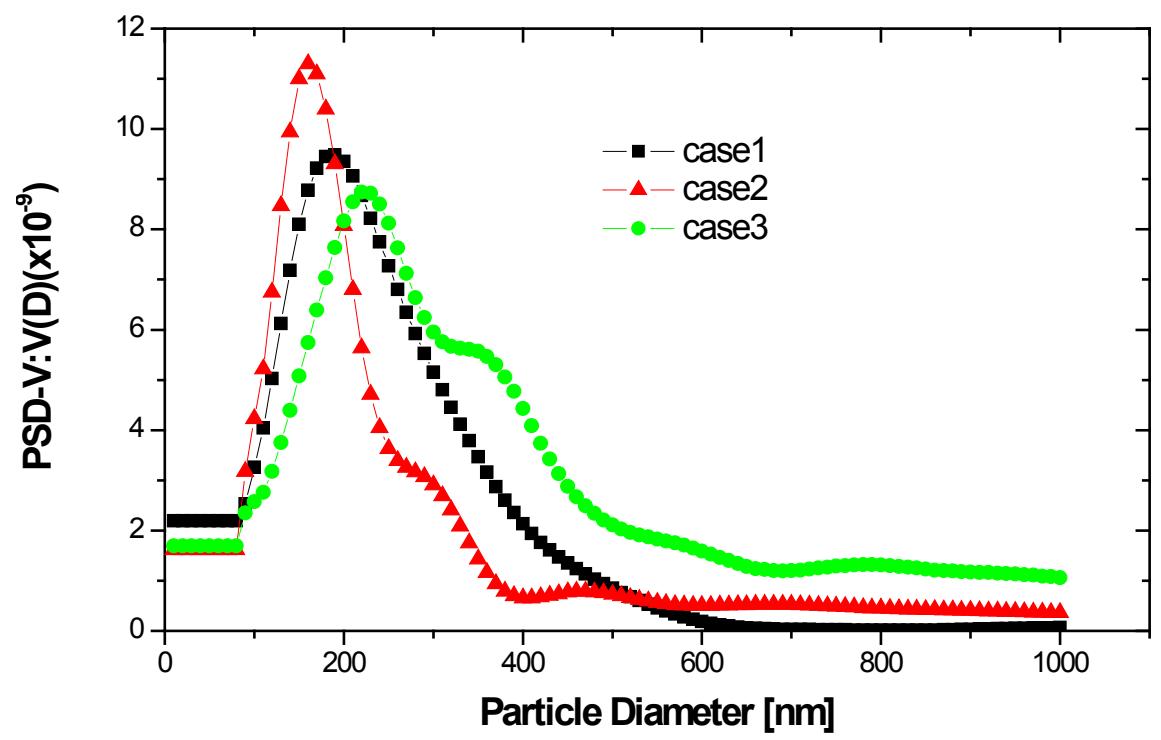

Figure 5. V-PSD obtained at $\Delta h_{1}=0 \mathrm{~cm}$ below the cathode and $\Delta t=160 \mathrm{~s}$ after the beginning of the $\mathrm{C}_{2} \mathrm{H}_{2}$ injection. The inversion procedure has been conducted successively using the 3 refractive indices reported on Table 1 .

Table 1. Summary of the various sources of optical indices used in the visible range.

\begin{tabular}{ccl}
\hline Refractive index & Reference & \multicolumn{1}{c}{ Synthesis process used } \\
\hline Case 1 & {$[23]$} & $\begin{array}{l}\text { combustion of a propane-oxygen mixture } \\
\text { plasma arc between two electrodes of amorphous } \\
\text { carbon in argon atmosphere (ACAR) } \\
\text { Case 2 }\end{array}$ \\
Case 3 & {$[17]$} & $\begin{array}{l}\text { plasma arc between two electrodes of amorphous } \\
\text { carbon in hydrogen atmosphere }\left(\mathrm{ACH}_{2}\right)\end{array}$ \\
\hline
\end{tabular}

Table 2. Concentration in volume AT $\Delta H_{1}=0 \mathrm{~cm}$ below the cathode and $\Delta T=160 \mathrm{~s}$ after the beginning of the $\mathrm{C}_{2} \mathrm{H}_{2}$ injection.

\begin{tabular}{cc}
\hline Refractive index & Concentration in volume: $\mathrm{C}_{\mathrm{v}}\left[\times 10^{-6}\right]$ \\
\hline Case 1 & 2.23 \\
Case 2 & 1.98 \\
Case 3 & 2.98 \\
\hline
\end{tabular}

\section{Conclusions}

We have studied the in-situ particle size distribution of the dust obtained by a low pressure Argon acetylene mixture RF discharge, using a commonly known method: inverse method accompanied by a regularization of Tikhonov which allows us to find with a good precision the distributions in particle size responsible for extinguishing the light beam used as a non-intrusive diagnostic means.

The appearance of small particles, generally less than $100 \mathrm{~nm}$, is frequently observed in the particle size distributions found by the reverse method. These particles should be ignored when using the results.

The reverse method has been applied to experimental data. This allowed on 
the one hand to test the influence of the distance of the light beam with respect to the cathode of the reactor and the time of discharge on the distribution of the dust. The results obtained are compatible with the experimental measurements of transmittance rate and on the other hand to test the influence of the choice of optical indices used for the calculation of the radiative properties of the particles. In this case the results provided by the inverse method depend strongly on the choice of optical indices used. Despite this, the trends observed during the evolution of the particle population are qualitatively similar. The choice of optical index 3rd case of Table 2 seems to provide particle sizes close to those found in the literature or well by experimental measurements using Scanning Electron Microscope (MEB).

In order to obtain information on the particle size distribution of the nanoscale dust cloud, we have chosen a technique of inverse computation by using experimental measurements of the transmittance obtained by a spectrometer and to be based on the hypothesis of spherical particles (theory of Mie). We found that the PSD-V is the lowest closest to the cathode, which indicates that the density of powders is greater when approaching the cathode; this shows that the powders remain in the vicinity immediate result of the cathode, and in good agreement with the measurements of the transmittance obtained in the spectral range of between $300 \mathrm{~nm}$ and $1000 \mathrm{~nm}$.

Since we do not have a precise knowledge of the optical indices of dust, we have studied the influence of the choice of optical indices used on the results; in this sense the inversion procedure has been applied to experimental data using the radiative properties calculated using 3 sources of different optical indices; the result that we have deduced is that the less the material is composed of absorbing particles, the lower the volume fraction.

The permanent interaction between the digital aspect and the experimental aspect will eventually lead to a more complete understanding of the agglomeration phenomenon. Possessing a tool to quantitatively monitor the size distribution of powders from non-intrusive optical diagnostics and easily implantable in a reduced environment is an important feature for a better implementation on tokamaks, including the future ITER reactor.

\section{Conflicts of Interest}

The authors declare no conflicts of interest regarding the publication of this paper.

\section{References}

[1] Kovacevic, E., et al. (2005) A Candidate Analog for Carbonaceous Interstellar Dust: Formation by Reactive Plasma Polymerization. The Astrophysical Journal, 623, 242-251. https://doi.org/10.1086/428392

[2] Sciamma-O’Brien, E., et al. (2010) Titan's Atmosphere: An Optimal Gas Mixture for Aerosol Production. Icarus, 209, 704-714. https://doi.org/10.1016/j.icarus.2010.04.009

[3] Selwyn, G.S., et al. (1989) In Situ Laser Diagnostic Studies of Plasma-Generated 
Particulate Contamination. Journal of Vacuum Science \& Technology A, 7, 2758-2765.

[4] El Kebch, A., Dlimi, N., Saifaoui, D., Dezairi, A. and El Mouden, M. (2016) Modelling and Simulation of Physical Sputtering. Molecular Crystals and Liquid Crystals, 627, 183-189. https://doi.org/10.1080/15421406.2015.1137676

[5] Federici, G. (2001) Plasma-Material Interactions in Current Tokamaks and Their Implications for Next Step Fusion Reactors. Nuclear Fusion, 41, 1967-2137. https://doi.org/10.1088/0029-5515/41/12/218

[6] Mitteau, R., et al. (2006) Evaluation of the Growth of Carbonaceous Deposit in Steady State Tore Supra Using Infrared Thermography. Nuclear Fusion, 46, S49-S55. https://doi.org/10.1088/0029-5515/46/3/S07

[7] Bardin, S. (2012) Etude des Intéraction Plasma-Paroi par imagerie rapide: Applications au plasma de laboratoire et de tokamak. PhD Thesis, Université de Lorraine, Lorraine.

[8] Dlimi, N., El Kebch, A., Saifaoui, D., Dezairi, A. and El Mouden, M. (2014) Modeling and Simulation of the Agglomeration of Carbonaceous Dust in a Radio Frequency Discharge Using the Monte Carlo Technique. Optical and Quantum Electronics, 46, 47-56. https://doi.org/10.1007/s11082-013-9694-0

[9] Mishchenco, M.I., Travis, L.D. and Lacis, A.A. (2002) Scattering, Absorption, and Emission of Light by Small Particles, NASA.

[10] Bohren, C.F. and Huffman, D.R. (1998) Absorption and Scattering of Light by Small Particles. John Wiley \& Sons, New York. https://doi.org/10.1002/9783527618156

[11] Viera, G. and Box, M.A. (1985) Information Content Analysis of Aerosol Remote Sensing Experiments Using an Analytic Eigenfunction Theory: Anomalous Diffraction Pproximation. Applied Optics, 24, 4525-4533.

https://doi.org/10.1364/AO.24.004525

[12] Kourti, T., MacGregor, J.F. and Hamielec, A.E. (1991) Turbidimetric Techniques-Capability to Provide the Full Particle Size Distribution. In: Particle Size Distribution II, ACS Symposium Series, American Chemical Society, Washington DC, Vol. 472, 2-19. https://doi.org/10.1021/bk-1991-0472.ch001

[13] Dellago, C. and Horvath, H. (1993) On the Accuracy of the Size Distribution Information Obtained from Light Extinction and Scattering Measurements I. Basic Considerations and Models. Journal of Aerosol Science, 24, 129-141. https://doi.org/10.1016/0021-8502(93)90053-C

[14] Onofri, F.R.A., Barbosa, S., Wozniak, M., Toure, O. and Mroczka, J. (2010) Optical Characterization of Nanoparticles and Aggregates: Light Extinction Spectrometry. 15th IntSymp on Applications of Laser Techniques to Fluid Mechanics, Lisbon, 5-8 July 2010.

[15] Peng, Y. (2009) Synthèse et caractérisation de poussières carbonées dans une décharge radio-fréquence. $\mathrm{PhD}$ Thesis, Faculté des Sciences et Techniques, Institut Jean Lamour.

[16] Xu, F. (2007) Diffusion d'un faisceau quelconque par un sphéroïde et mesure en ligne de la vapeur humide par l'extinction spectrale de la lumière. $\mathrm{PhD}$ Thesis, University of Rouen, Mont-Saint-Aignan.

[17] Zubko, V.G., et al. (1996) Optical Constants of Cosmic Carbon Analogue Grains I. Simulation of Clustering by a Modified Continuous Distribution of Ellipsoids. Monthly Notices of the Royal Astronomical Society, 282, 1321-1329.

https://doi.org/10.1093/mnras/282.4.1321 
[18] Bohren, C.F., et al. (1983) Absorption and Scattering of Light by Small Particles. John Wiley and Sons, New York.

[19] Lawson, C.L. and Hanson, R.J. (1974) Solving Least Squares Problems. Prentice-Hall, Englewood Cliffs.

[20] Tikhonov, A.N., et al. (1995) Numerical Methods for the Solution of Ill-Posed Problems. K.A. Publishers, New York. https://doi.org/10.1007/978-94-015-8480-7

[21] Hansen, P.C. (1994) Regularization Tools: A Matlab Package for Analysis and Solution of Discrete Ill-Posed Problems. Numerical Algorithms, 6, 1-35. https://doi.org/10.1007/BF02149761

[22] Hansen, P.C. (2007) Regularization Tools Version 4.0 for Matlab 7.3. Numerical Algorithms, 46, 189-194. https://doi.org/10.1007/s11075-007-9136-9

[23] Chang, H. and Charalampopoulos, T.T. (1990) Determination of the Wavelength Dependence of Refractive Indices of Flame Soot. Proceedings of the Royal Society of London. Series A, 430, 577-591. https://doi.org/10.1098/rspa.1990.0107 